\title{
GENERALIZED FINITE DIFFERENCE SCHEMES WITH HIGHER ORDER WHITNEY FORMS
}

\author{
Lauri Kettunen*, Jonni Lohi, Jukka RÄBinä, Sanna Mönkölä And Tuomo Rossi
}

\begin{abstract}
Finite difference kind of schemes are popular in approximating wave propagation problems in finite dimensional spaces. While Yee's original paper on the finite difference method is already from the sixties, mathematically there still remains questions which are not yet satisfactorily covered. In this paper, we address two issues of this kind. Firstly, in the literature Yee's scheme is constructed separately for each particular type of wave problem. Here, we explicitly generalize the Yee scheme to a class of wave problems that covers at large physics field theories. For this we introduce Yee's scheme for all problems of a class characterised on a Minkowski manifold by (i) a pair of first order partial differential equations and by (ii) a constitutive relation that couple the differential equations with a Hodge relation. In addition, we introduce a strategy to systematically exploit higher order Whitney elements in Yee-like approaches. This makes higher order interpolation possible both in time and space. For this, we show that Yee-like schemes preserve the local character of the Hodge relation, which is to say, the constitutive laws become imposed on a finite set of points instead of on all ordinary points of space. As a result, the usage of higher order Whitney forms does not compel to change the actual solution process at all. This is demonstrated with a simple example.
\end{abstract}

Mathematics Subject Classification. 35L05, 35L10, 58G16, 58G20, 58 G40.

Received August 20, 2020. Accepted May 22, 2021.

\section{INTRODUCTION}

Finite difference kind of schemes introduced by Yee in 1966 [41] are commonly employed to approximate wave propagation problems in finite dimensional spaces. The popularity arises from the explicit character of the solution process; assuming linear and isotropic material, at each time step solving systems of equations reduces to inverting diagonal matrices. This makes the approach highly efficient lending itself also to parallel computing ${ }^{1}$. The textbook explanation makes it also rather easy to comprehend finite difference schemes, and likely, this is another reason for their popularity.

At the first place Yee introduced his approach to the analysis of electromagnetic waves. Thereafter the scheme has been extended one by one to other fields of physics. The fundamental idea of science is, however, not only to work out collections of specific cases, but also to generate a comprehension from which all particular cases can

\footnotetext{
Keywords and phrases. Finite difference method, whitney forms, differential geometry, differential forms, vector-valued forms, co-vector valued forms, electromagnetism, elasticity.

1 University of Jyväskylä, Faculty of Information Technology, University of Jyväskylä, 35, FI-40014 Jyväskylä, Finland.

*Corresponding author: lauri.y.o.kettunen@jyu.fi

${ }^{1}$ For instance, in [31] the authors have demonstrated solving problems with $10^{10}$ degrees of freedom.
} 
be derived when needed. Such a unifying view is also a key to enable software systems that are not restricted to specific application fields.

The formal background of Yee-like schemes -such as the finite integration technique [38], Tonti's cell method $[36,37]$, the "geometric approach" $[15,17,35]$ or Hirani's discrete exterior calculus [24] - is naturally presented in terms of differential geometry and chain complexes as explained by Bossavit in a series of papers [5-14]. The key issue is, unlike classical vector analysis differential geometry makes it possible to distinct between the differentiable and metric structure. To establish differential equations only the differentiable structure, that provides one with sufficient smoothness, is needed. The constitutive relations call for the metric structure instead. In case of Yee-schemes this implies, the differential equations can be imposed exactly in finite dimensional spaces whereas the constitutive laws cannot. This leads to the issue of the "discrete Hodge" [34] and to mass matrix diagonalization techniques that make to it possible to bypass Yee's original assumption of cartesian grids $[17,20,27]$. In addition, the formal background reveals the relation between the finite difference and the finite element methods $[4,18,19]$.

Our aim here is to introduce a framework from which "all" specific wave problems and the corresponding Yee-like schemes can be derived. For this we need a starting point general enough. This is obtained by imposing a pair of first order differential equations,

$$
\mathrm{d} F=0 \quad \text { and } \quad \mathrm{d} H=G
$$

and a constitutive law,

$$
H=\star F,
$$

in space-time, that is, on a Minkowski manifold. Here, the differential operator d is either the exterior derivative or the so-called exterior covariant derivative, and $\star$ is the Hodge operator in space-time. This generalized wave problem corresponds to a class of wave problems covering physical field theories at large.

To work out specific wave problems such as to electromagnetism or elasticity, we assume next a decomposition of space-time into space and time and express the generalized wave problem, i.e., equation (7.4) in Section 7, in $(n+1)$-dimensional spaces. The well-known field theories of physics are then found as instances of this generalized "continuous level" wave problem.

Thereafter we introduce the Yee-scheme to this generalized wave problem. For this finite dimensional problem we assume both in space and time a pair of cell complexes dual to each other to systematically construct the corresponding differential operators. Thereafter we show that Yee-like schemes can be interpreted to impose the constitutive laws at a finite amount of points instead of at all ordinary points of space. This sums up to say, the approximation involved in Yee-likes schemes consists of imposing differential equations in finite dimensional spaces and of satisfying constitutive laws at a finite amount of points.

The latter interpretation that the constitutive laws hold only at a finite amount of points is significant because it separates the issue of interpolating fields with higher order elements completely from the solution process. In other words, when establishing the system of equations there is no need to take sides on any kind of elements. That is, setting up the system of equations does not require any elements. Consequently, this legitimates to consider the choice of elements as a post processing question. We would, however, like to stress out, this is not the only possible view on the usage of higher order elements.

In the last part of the paper we explain the usage of higher order Whitney elements in the post processing stage. This is also demonstrated with an example from electromagnetism. Up to this point we assume a dual pair of cell complexes, but the usage of higher order Whitney forms sets an additional condition of a simplicial primal mesh. 


\section{BASIC ASSUMPTIONS AND PRELIMINARIES}

In the next section we introduce briefly the required formal background from mathematics. A reader not that interested in the technical details may jump directly to the generalized wave problem to equation (7.4) in Section 7.

To model space-time, we assume a Minkowski manifold $\Omega$ with metric tensor $g$. In addition, we assume a decomposition of space-time into space $\Omega_{s}$ and time $\Omega_{t}$. In other words, we have $\Omega=\Omega_{t} \times \Omega_{s}$. The dimension of $\Omega$ is $(1+n)=4$, and the signature is assumed to be $(-,+,+,+)$.

We denote the tangent vector space at point $x \in \Omega$ by $T_{x} \Omega$. The elements of $T_{x} \Omega$ are the tangent vectors of all trajectories through point $x^{2}$. The dual space $T_{x}^{*} \Omega$ of $T_{x} \Omega$ is obtained by pairing each vector $v \in T_{x} \Omega$ with a linear map $\nu \in T_{x} \Omega \rightarrow \mathbb{R}$ such that

$$
\nu(v)=(u, v)=u \cdot v
$$

holds for all $u \in T_{x} \Omega$. The elements of the dual space $T_{x}^{*} \Omega$ are called covectors.

Tangent vectors are naturally extended to $p$-vectors, $p=0, \ldots, n$, with the exterior algebra $\bigwedge^{p} T_{x} \Omega$ generated using (the addition and scalar multiplication of $\wedge^{p} T_{x} \Omega$ and) the exterior product $\wedge$. The elements of the dual space $\bigwedge^{p} T_{x}^{*} \Omega$ are $p$-covectors, which map $p$-vectors of $\bigwedge^{p} T_{x} \Omega$ to reals.

\section{ORDINARY AND E-VALUED DIFFERENTIAL FORMS}

Differential forms of degree $p$, or, in other words, (ordinary) $p$-forms are $p$-covector fields. At each point of $\Omega$ a $p$-form maps $p$-vectors to real numbers, such as in electromagnetism the magnetic flux is a 2 -form which maps in the virtual neighbourhoods of each point of space 2-vectors (i.e., virtually small oriented "patches") to real numbers that represent the flux.

Suppose next $E$ is a smooth real vector bundle over $\Omega$. An $E$-valued differential form of degree $p$ is a section of $E \otimes \bigwedge^{p} T^{*} \Omega[2,22,23]$. Vector-valued and covector-valued forms are obtained when $E$ is chosen to be $T \Omega$ and $T^{*} \Omega$, respectively.

This is to say, having vector field $e$ and $p$-form $f, e \otimes f$ is a vector-valued differential $p$-form, which depends linearly on each factor $e$ and $f$.

In more detail, in local coordinates, if $e$ and $f$ are given by $e=\left[e_{x}, e_{y}, e_{z}\right]^{T}$ and $f=f_{x} \mathrm{~d} x+f_{y} \mathrm{~d} y+f_{z} \mathrm{~d} z$, then the corresponding vector-valued form $e \otimes f$ can be written by

$$
e \otimes f=\left[e_{x} f, e_{y} f, e_{z} f\right]^{T} .
$$

Furthermore, when $e \otimes f$ acts on vector field $v$, the result is

$$
(e \otimes f)(v)=e \otimes f(v)=\left[e_{x} f(v), e_{y} f(v), e_{z} f(v)\right]^{T}=f(v)\left[e_{x}, e_{y}, e_{z}\right]^{T},
$$

where $f(v)$ is a scalar field of reals.

For covector-valued differential forms of degree $p$, say, $\omega=\omega_{x} \mathrm{~d} x+\omega_{y} \mathrm{~d} y+\omega_{z} \mathrm{~d} z$ is a covector field and 1-form $f$ is given by $f=f_{x} \mathrm{~d} x+f_{y} \mathrm{~d} y+f_{z} \mathrm{~d} z$. Then, a covector-valued 1-form

$$
\omega \otimes f=\omega_{x} \mathrm{~d} x \otimes f+\omega_{y} \mathrm{~d} y \otimes f+\omega_{z} \mathrm{~d} z \otimes f,
$$

and when this acts on vector $v$ field, it yields

$$
(\omega \otimes f)(v)=\omega \otimes f(v)=f(v)\left(\omega_{x} \mathrm{~d} x+\omega_{y} \mathrm{~d} y+\omega_{z} \mathrm{~d} z\right) .
$$

\footnotetext{
${ }^{2}$ In case of Euclidean spaces, the tangent spaces $T_{x} \Omega$ and $T_{y} \Omega$ of any two points $x$ and $y$ are canonically identified with each other. For this reason, in the Euclidean setting the existence of the tangent spaces is not usually explicitly considered.
} 


\section{HODGE OPERATOR}

The Hodge operator $\star[3,21,22,25]$ maps ordinary $p$-forms to $(n-p)$-forms. It is defined with the Riesz representation theorem [42]:

Definition 4.1. A linear map from $p$-vectors to $(n-p)$-vectors is the Hodge operator, if for all $p$-vectors $u$ and $v$ the image $\star v$ of $v$ satisfies

$$
u \wedge \star v=(u, v) \omega_{0},
$$

where $\omega_{0}$ is the unit $n$-vector.

The Hodge operator for $p$-covectors is defined similarly; in above vectors are just replaced with covectors. Notice, the Hodge operator, or more precisely, the values of $\star v$ in the chosen frame depend on the metric.

Remark 4.2. The Hodge star is a local operator between tangent spaces of a point. While the metric tensor may have some continuity properties, at an observation point $x$ the values of the metric tensor at the neighbouring points have no effect at $x$.

Next, let us extend the Hodge operator to vector and covector-valued forms. For this, we need the flat and sharp map between sections $\Gamma(T \Omega)$ and $\Gamma\left(T^{*} \Omega\right)$. (In classical analysis, such sections correspond with vector and covector fields.) The flat map is given by $b: \Gamma(T \Omega) \rightarrow \Gamma\left(T^{*} \Omega\right), e \mapsto \mathrm{i}_{e} g=g(e, \cdot)$, where $\mathrm{i}_{e}$ is the contraction of $g$ by $e^{3}$. The sharp map $\sharp: \Gamma\left(T^{*} \Omega\right) \rightarrow \Gamma(T \Omega)$ is the inverse of this.

Now the extension of the Hodge operator to vector and covector-valued forms is defined as follows.

Definition 4.3. A linear map between vector and covector-valued forms is the extension of the Hodge operator, if it satisfies

$$
\begin{aligned}
& \star: \Gamma\left(T \Omega \otimes \bigwedge^{p} T^{*} \Omega\right) \rightarrow \Gamma\left(T^{*} \Omega \otimes \bigwedge^{n-p} T^{*} \Omega\right), s \otimes \sigma \mapsto b s \otimes \star \sigma, \\
& \star: \Gamma\left(T^{*} \Omega \bigwedge^{p} T^{*} \Omega\right) \rightarrow \Gamma\left(T \Omega \otimes \bigwedge^{n-p} T^{*} \Omega\right), s \otimes \sigma \mapsto \sharp s \otimes \star \sigma .
\end{aligned}
$$

Accordingly, the Hodge operator maps a vector-valued form to a covector-valued form and vice versa.

\section{Formal SUM OF FIELDS}

Our aim is to find a class of field problems to which Yee-like schemes can be applied. Accordingly, we are not interested in $p$-forms of a particular degree, but instead, in all degrees $p=0, \ldots, n$. To "hide" the information of the degree, we form a formal sum $F$ of ordinary differential forms with real coefficients:

$$
F=\alpha_{0} f^{0}+\alpha_{1} f^{1}+\cdots+\alpha_{n} f^{n} \in \bigoplus_{p=0}^{n} \bigwedge^{p} T^{*} \Omega,
$$

where $\alpha_{0}, \ldots, \alpha_{n} \in \mathbb{R}$. This implies, any $p$-form from any physics field theory is an instance of $F$, and conversely, by operating with $F$ we can cover at once all fields expressible with ordinary differential forms.

In the same manner we form also formal sums of vector and covector-valued forms:

$$
F=\alpha_{0} f^{0}+\alpha_{1} f^{1}+\cdots+\alpha_{n} f^{n} \in \bigoplus_{p=0}^{n}\left(E \otimes \bigwedge^{p} T^{*} \Omega\right),
$$

where $\alpha_{0}, \ldots, \alpha_{n} \in \mathbb{R}$ and $E$ is either $T \Omega$ or $T^{*} \Omega$. Accordingly, any vector or covector-valued form becomes an instance of $F$.

\footnotetext{
${ }^{3}$ The metric tensor returns a real number for a pair of vectors. The contraction by $e$ fills the other slot of $g$ resulting in a linear function on vectors.
} 


\section{Differentiation}

Ordinary differential forms are differentiated with the exterior derivative d. To differentiate vector and covector valued forms, one needs the so called exterior covariant derivative $\mathrm{d}_{\nabla}$, where $\nabla$ is the connection $[2,3,22]$ :

$$
\mathrm{d}_{\nabla}(s \otimes f)=\mathrm{d}_{\nabla} s \wedge f+s \otimes \mathrm{d} f .
$$

From now on, to simplify the notation and to focus on the main issues behind generalizing Yee-like shemes, we assume $\Omega_{s}$ is a bounded domain of Euclidean space. This implies a trivial connection. Consequently, in this case we have $\mathrm{d}_{\nabla}(s \otimes f)=s \otimes \mathrm{d} f$, and $\mathrm{d}_{\nabla}$ can be identified as component-wise differentiation with d. Accordingly, symbol d will be employed to denote both to the exterior covariant and the exterior derivative.

\section{Generalized WaVe propagation problem}

To construct the generalized wave problem, we start from the solutions of

$$
\mathrm{d} F=0 \quad \text { and } \quad \star \mathrm{d} \star F=\star \star G,
$$

in Minkowski space. Here, $G$ is the source term, and $\star$ is the Hodge operator in space-time. The $\star \star$ operator is up to the sign equal to one, $\star \star=(-1)^{p(n-p)+1}$.

To express these differential equations in space and time, notice first that any differential form $f^{p}$ on $\Omega_{t} \times \Omega_{s}$ can be decomposed into a time -like and space-like component as follows

$$
f^{p}=f_{t}^{p}+f_{s}^{p}=\mathrm{d} t \wedge f_{*}^{p-1}+f_{s}^{p} .
$$

Consequently, in dimension $n=1+3$ any general field $F$ can be written as a formal sum

$$
\begin{aligned}
F & =f^{0}+f^{1}+f^{2}+f^{3}+f^{4} \\
& =f^{0}+\left(\mathrm{d} t \wedge f_{*}^{0}+f_{s}^{1}\right)+\left(\mathrm{d} t \wedge f_{*}^{1}+f_{s}^{2}\right)+\left(\mathrm{d} t \wedge f_{*}^{2}+f_{s}^{3}\right)+\mathrm{d} t \wedge f_{*}^{3} .
\end{aligned}
$$

In space and time the exterior (covariant) derivative splits into $\mathrm{d}=\mathrm{d} t \wedge \partial_{t}+\mathrm{d}_{s}$, where $\mathrm{d}_{s}$ is the derivative in spatial space.

The Hodge operator in spatial space is denoted by $\star_{s}$ and in time by $\star_{t}$. By definition, $\star_{t}$ should satisfy ${ }^{4}$

$$
\star_{t} 1=\mathrm{d} t \quad \text { and } \quad \star_{t} \mathrm{~d} t=1 .
$$

Remark 7.1. Both $\star_{s}$ and $\star_{t}$ follow from the Hodge operator in Minkowski space once space-time is decomposed into space and time. The literature of finite differences and finite elements has not recognized this, and consequently, the focus has solely been on the Hodge operator in spatial space. However, the Hodge operator in time is as significant as $\star_{s}$, and the explicit recognition of $\star_{t}$ is a key in extending finite differences into higher order not only in space but also in time.

For brevity, from now on, we will denote the space-like exterior derivative and Hodge operator by $\mathrm{d}$ and $\star$, respectively. As shown in [28], in space and time and $n=1+3$, the pair of differential equations in (7.1) is

\footnotetext{
${ }^{4}$ Notice, that although in Minkowski space one has $(\mathrm{d} t, \mathrm{~d} t)=-1$, the 1-dimensional space of time is Euclidean, and consequently, we have $(\mathrm{d} t, \mathrm{~d} t)=1$.
} 
equivalent to

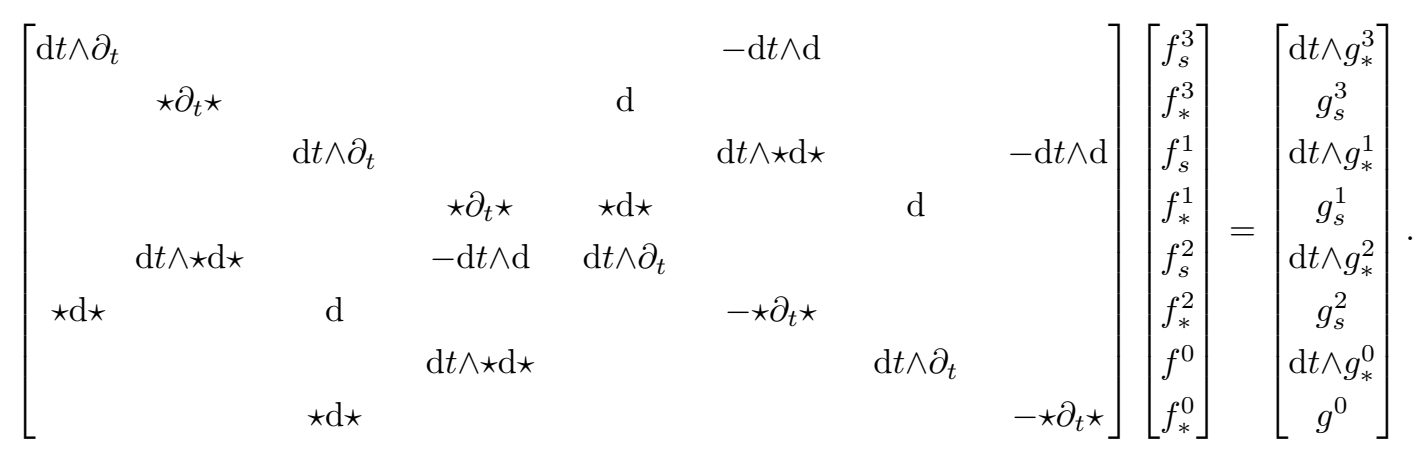

Particular field theories, such as electromagnetism or elasticity, are instances of this generalized setting. For example, Maxwell's equations are instantiated by choosing $F=e \wedge \mathrm{d} t+b=-\mathrm{d} t \wedge e+b$ and $G=\star j-\mathrm{d} t \wedge \star q$, where electric field strength $e$ is a 1-form, magnetic flux $b$ and current $j$ are 2-forms, and charge $q$ a 3-form. When $F$ and $G$-that is, $f_{*}^{1}=-e, f_{s}^{2}=b, g_{s}^{1}=\star j, g_{*}^{0}=-\star q$, all the other entries are null- are substituted to the generalized system, the second, fifth, fourth, and seventh row yields

$$
\begin{aligned}
& \mathrm{d} b=0, \quad \mathrm{~d} b=0, \\
& \mathrm{~d} t \wedge \mathrm{d} e+\mathrm{d} t \wedge \partial_{t} b=0, \\
& \mathrm{~d} e+\partial_{t} b=0 \\
& -\star \partial_{t} \star_{\epsilon} e+\star \mathrm{d} \star_{\nu} b=\star j, \\
& \Leftrightarrow \\
& -\partial_{t} \star_{\epsilon} e+\mathrm{d} \star_{\nu} b=j \\
& -\mathrm{d} t \wedge \star \mathrm{d} \star_{\epsilon} e=-\mathrm{d} t \wedge \star q, \\
& \mathrm{~d} \star_{\epsilon} e=q,
\end{aligned}
$$

respectively. The material parameters can be incorporated to the Hodge operator [16], and accordingly, we have embedded permittivity $\epsilon$ and permeability $\mu=1 / \nu$ into $\star_{\epsilon}$ and $\star_{\nu}$.

To find the differential equations for small-strain elasticity [1] with linear and isotropic materials, we model displacement $\nu$ as a vector valued 0 -form. Its time derivative is velocity $u=\partial_{t} \nu$. Mass density is denoted by $\rho$, and $f_{v}$ is the body force. Stress $\sigma$ is a covector-valued 2-form, and linearised strain $\varepsilon$ is a vector-valued 1-form $[26,33,40]$. The stress-strain relation describing the elastic properties of the medium (i.e., material) can be incorporated into the Hodge operator $\star_{s}^{C}$, and consequently, we employ superscript $C$ to denote this. [28,29]

The differential equations of small-strain elasticity are obtained by choosing $f_{*}^{0}=u, f_{s}^{1}=\varepsilon$, and $g^{0}=-\star_{s} f_{v}$ [28]. Substituting these back to the generalized system yields the following first order differential equations for small-strain elasticity

$$
\begin{aligned}
-\partial_{t} \varepsilon+\mathrm{d} u & =0, & \varepsilon & =\mathrm{d} \nu, \\
\star_{s}^{\rho} \partial_{t} u-\mathrm{d} \sigma & =f_{v}, & u & =\partial_{t} \nu .
\end{aligned} \quad \sigma=\star_{s}^{C} \varepsilon,
$$

\section{Finite dimensional COUnterpart of the WAVE PRoblem}

Next, our aim is to construct a finite dimensional counterpart to (7.4). For this, we want to map the formal sums of differential forms (5.1) and (5.2), to finite dimensional vector spaces. Furthermore, for the finite dimensional model we need also to map $d$ and $\star$ to linear operators between such vector spaces so that key properties are preserved.

\subsection{Chains and cochains}

To construct the finite dimensional model we first define a regular cell complex $T$ as a triangulation of an $n$-dimensional manifold $M$. For this we need first a $p$-cell $\sigma^{p}, p=0, \ldots, n$. It is a diffeomorphic map from an oriented $p$-dimensional convex polyhedral cell $s$ in $\mathbb{R}^{n}$ to $M$. 


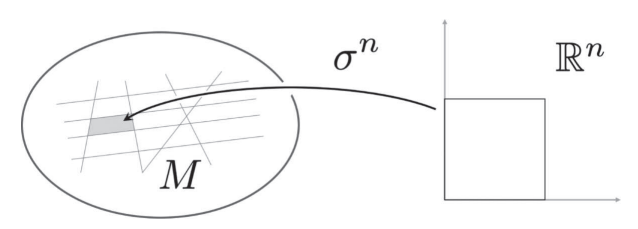

Figure 1. Mappings of oriented $p$-cells form a regular cell complex $T$ triangulating manifold $M$.

Definition 8.1. A finite set $T$ of cells is a regular cell complex,

(1) if $\sigma(s) \in T$, then each face of $\sigma(s)$ is a cell of $T$,

(2) no interior of $\sigma(s)=\sigma(s)-\partial \sigma(s)$ intersects images of cells of lower dimension of $T$, and

(3) each intersection $\sigma(s) \cap \sigma^{\prime}(s)$, if not empty, is the image of an $s \in \mathbb{R}^{n}$.

Definition 8.2. A regular cell complex $T$ is a triangulation of an $n$-dimensional manifold $M$, if $\bigcup_{\sigma^{n} \in T} \sigma^{n}(s)=$ $M$.

The formal sums of $p$-cells with real coefficients

$$
c=\sum_{i} \alpha_{i} \sigma_{i}^{p}, \alpha_{i} \in \mathbb{R}, \sigma_{i}^{p} \in T^{p}
$$

are called (algebraic) $p$-chains.

Let $C^{p}$ be the linear space of $p$-chains of $T$. The elements of the conjugate space $C_{p}$ of linear functionals are called (algebraic) $p$-cochains; if $X$ is a $p$-cochain and $c$ a $p$-chain, then $X$ maps $c$ to $X(c) \in \mathbb{R}$.

Our aim is to construct a map from the space of differential forms to some finite dimensional vector spaces. For this purpose, we exploit chains and cochains to define the de Rham map:

Definition 8.3. Map $\mathcal{C}$ from differential forms to cochains is the de Rham map between differential forms and cochains, if for all $p$-chains $c \in C^{p}$ and all $p$-forms $f$ on the manifold map $\mathcal{C}$ satisfies

$$
\mathcal{C} f: C^{p} \rightarrow \mathbb{R}, \quad c \mapsto \int_{c} f .
$$

The de Rham map provides us with a tool to map differential forms on the manifold to the finite dimensional spaces of cochains on $T$.

\subsection{Coboundary -the finite dimensional counterpart of $d$}

Next, we construct explicitly a linear operator as the finite dimensional counterpart of the exterior derivative $\mathrm{d}$ so that its key properties are retained.

For this, recall first that the exterior derivative is defined such that the Stokes theorem holds; the integral of $\mathrm{d} f$ over a $(p+1)$-dimensional oriented manifold $\omega$ should equal to the integral of $f$ over the boundary of $\omega$,

$$
\int_{\omega} \mathrm{d} f=\int_{\partial \omega} f .
$$

We employ the Stokes theorem to define the coboundary operator:

Definition 8.4. Function d : $C_{p} \rightarrow C_{p+1}$ is the coboundary, if for all $X \in C_{p}$ and $c \in C^{p+1}$

$$
\mathrm{d} X(c)=X(\partial c)
$$

holds. 
The definition implies, the coboundary share the following properties with the exterior derivative:

(1) The coboundary is a linear operator,

(2) it increases the grade $p$ by one, and

(3) it fulfils dd $\equiv 0$.

The first two properties follows from the definition, and the last property from: For all $(X, c) \in C_{p} \times C^{p+2}$ ones has

since $\partial \partial \equiv 0$.

$$
\operatorname{dd} X(c)=\mathrm{d} X(\partial c)=X(\partial \partial c) \equiv 0
$$

Corollary 8.5. The definition of the coboundary implies operators $\mathrm{d}$ and $\mathcal{C}$ commute:

$$
\mathrm{d} \mathcal{C}=\mathcal{C} \mathrm{d}
$$

This is to say the following diagram

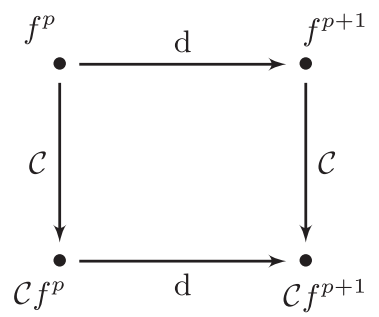

is commutative.

Proof. Let $f$ be a $p$-form. The Stokes theorem implies, for all $p$-forms $f$ and $(p+1)$-chains $c$ we have

$$
\mathcal{C} \mathrm{d} f(c)=\mathcal{C} f(\partial c)=\mathrm{d} \mathcal{C} f(c) .
$$

Remark 8.6. All what is presented above on chains, cochains, and on the coboundary applies as well to tilings of the 1-dimensional time-like manifold $\Omega_{t}$ and to time derivative $\mathrm{d} t \wedge \partial_{t}$, which coincides with the exterior derivative in $\Omega_{t}$.

\subsection{Hodge relation in the finite dimensional case}

The Hodge operator maps $p$-vectors to $(n-p)$-vectors and $p$-covectors to $(n-p)$-covectors at each point of a manifold. As chains or cochains do not belong to the domain of $\star$, there exists no Hodge operator for them. This implies, we need a Hodge kind of operator to chains and cochains.

So, we seek for an operator that maps $p$-chains to $(n-p)$-chains, and for this, we identify first the $p$-cells of $T^{p}$ with $p$-vectors:

Definition 8.7. In $\mathbb{R}^{n}$ the $p$-vector $\{s\}$ of an oriented $p$-cell $s$ is the following triple: (i) the set of $p$-planes parallel to $s$, (ii) the orientation of $s$, and (iii) the $p$-dimensional volume $|s|$ of $s$.

Definition 8.8. At point $x$ of manifold $M$ the $p$-vector $\{\sigma\} \in T_{x}^{p} M$ of an oriented $p$-cell $\sigma(s)$ is the pushforward of $\{s\}$ by $\sigma$.

Next, given a manifold $M$, such as $\Omega_{t}$ or $\Omega_{s}$, we introduce for each $p$-vector $\left\{\sigma^{p}\right\} \in T_{x}^{p} M$ the set of perpendicular $(n-p)$-vectors; $\left\{\tilde{\sigma}^{n-p}\right\} \in T_{x}^{n-p} M$ is perpendicular to $\left\{\sigma^{p}\right\}$, if there exists an $\alpha \in \mathbb{R}-\{0\}$ such that

$$
\left\{\tilde{\sigma}^{n-p}\right\}=\alpha \star\left\{\sigma^{p}\right\}
$$

holds.

Exploiting such perpendicularity we introduce a cell complex dual to $T$ : 
Definition 8.9. A cell complex is the dual complex $\tilde{T}$ of triangulation $T$, if

(1) for each $p$-cell $\sigma^{p} \in T, p=0, \ldots n$, there exists a unique $(n-p)$-cell $\tilde{\sigma}^{n-p} \in \tilde{T}$ so that, images $\sigma^{p}(s)$ and $\tilde{\sigma}^{n-p}(s)$ intersect exactly at one point.

(2) The vectors $\left\{\sigma^{p}\right\} \in T_{x}^{p} M$ and $\left\{\tilde{\sigma}^{n-p}\right\} \in T_{x}^{n-p} M$ fulfil (8.1) at the intersection point $x$ of $\sigma^{p}(s)$ and $\tilde{\sigma}^{n-p}(s)$.

Remark 8.10. This definition does not yet guarantee the existence or uniqueness of the dual complex for all triangulations $T$. This corresponds with the well-known restriction, that the choice of the triangulation in finite difference kind of approaches is not as flexible as, for example, in case of the finite element method.

Remark 8.11. Since $\tilde{T}$ is a complex, it admits the coboundary operator.

From now on we will assume a pair of triangulations $(T, \tilde{T})$ whose $p$ and $(n-p)$-cells are indexed with elements of set $I$ such that every $i \in I$ corresponds to a pair of intersecting cells $\left(\sigma_{i}^{p}, \tilde{\sigma}_{i}^{n-p}\right) \in T^{p} \times \tilde{T}^{n-p}$. The orientation of $\left\{\sigma_{i}^{p}\right\} \wedge\left\{\tilde{\sigma}_{i}^{n-p}\right\}$ is denoted by $s g n$ and the space of $(n-p)$-chains of the dual complex by $\tilde{C}^{n-p}$.

Now, map

$$
H: C^{p} \rightarrow \tilde{C}^{n-p}, \quad c=\sum_{i} \alpha_{i} \frac{\sigma_{i}^{p}}{\left|\sigma_{i}^{p}\right|} \mapsto \tilde{c}=\operatorname{sgn} \sum_{i} \alpha_{i} \frac{\tilde{\sigma}_{i}^{n-p}}{\left|\tilde{\sigma}_{i}^{n-p}\right|}
$$

provides us with a Hodge kind of map we have been after. Map $H$ is akin to $\star$ in the sense that

(1) $H$ is linear,

(2) $H$ yields a unique counterpart in $\tilde{C}^{n-p}$ for each element of $C^{p}$,

(3) $H$ maps $p$-cells with unit $p$-volumes to perpendicular $(n-p)$-cells with unit $(n-p)$-volumes, and

(4) $H^{-1} H$ is an identity.

Consequently, map $H$ may well be called a "discrete Hodge" [34]. However, be aware, the name is a compound word as $H$ is not the Hodge map.

To extend $H$ to cochains, we start from the constitutive laws. A constitutive law between $p$-form $f$ and $(n-p)$-form $f^{\prime}$ is of the form

$$
f=\star f^{\prime} .
$$

This is equivalent to saying

$$
f(v)=f^{\prime}(\star v) \quad \forall v \in T_{x}^{p}
$$

hold at all points $x \in \Omega$.

Informally, for all pairs $\left(f, f^{\prime}\right)$ fulfilling (8.2) we have

$$
\mathcal{C} f \approx \mathcal{C} f^{\prime} H
$$

and this suggests a modelling decision: Given $p$-form $f$, we define cochain $F^{\prime} \in \tilde{C}_{n-p}$ such that

$$
\mathcal{C} f(c)=F^{\prime}(H c) \quad \forall c \in C^{p}
$$

holds. In other words, we specify $F^{\prime}$ as an approximate of $C f^{\prime}$ providing us with a counterpart (or proxy) of (8.3) in finite dimensional spaces.

Next, map $H$ is easily extended to cochains by defining a map $*: C_{p} \rightarrow \tilde{C}_{n-p}$ such that for all $X \in C_{p}$ we have

$$
* X: \tilde{C}^{n-p} \rightarrow \mathbb{R}, \quad \tilde{c} \mapsto X\left(H^{-1} \tilde{c}\right) .
$$

Consequently, operators $*^{-1} *$ and $*^{-1}$ become identities.

Notice, intuitively, for all pairs $\left(f, f^{\prime}\right)$ fulfilling (8.2) we have

$$
* \mathcal{C} f \approx \mathcal{C} f^{\prime} \quad \text { or } \quad * \mathcal{C} \approx \mathcal{C} \star .
$$


In more precise terms, the approximation is to say the following diagrams

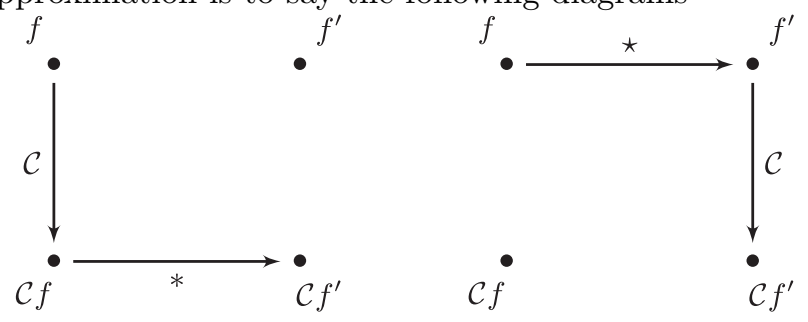

cannot be combined as a commutative diagram.

Remark 8.12. As an immediate consequence of (8.3) we have

$$
f\left(\left\{\sigma_{i}^{p}\right\} /\left|\left\{\sigma_{i}^{p}\right\}\right|\right)=\star f\left(\left\{\tilde{\sigma}_{i}^{n-p}\right\} / \mid\left\{\tilde{\sigma}_{i}^{n-p} \mid\right) \quad \forall i \in I .\right.
$$

This will provide us with a complementary view on the constitutive laws between finite dimensional spaces of cochains. The finite dimensional wave problem is about imposing the constitutive laws (i) at the (finite amount of) intersection points between $p$ and $(n-p)$-cells of $T$ and $\tilde{T}$, respectively, and (ii) with the $p$ and $(n-p)$-vectors that correspond to the intersection cells. That is, instead of (8.3), at each intersection point the constitutive law is imposed for a certain $p$ and $(n-p)$-vector pair.

\subsection{Finite dimensional wave problem}

We have now all what is needed to introduce the finite dimensional image of the generalized wave propagation problem (7.4). To map the operators and the differential forms involved in (7.4) to finite dimensional spaces, we assume a pair of dual complexes $T_{t}$ and $T_{s}$ in time and space, respectively, that tile $\Omega_{t}$ and $\Omega_{s}$. In addition, we denote the de Rham map in time by $\mathcal{C}_{t}$ and in the spatial space by $\mathcal{C}_{s}$.

Let us start from the operators:

(1) $\mathrm{d}$ is the exterior derivative, and in the finite dimensional case it is mapped to the coboundary operator.

$(2) \star d \star$ is up to the sign the adjoint $\delta$ of $\mathrm{d}$. As the Hodge operator for differential forms is mapped to $*$ and $\mathrm{d}$ maps to the coboundary operator, the image of $\star d \star$ is $*^{-1} \mathrm{~d} *$.

(3) Operator $\mathrm{d} t \wedge \partial_{t}$ is the product of 1 -form $\mathrm{d} t$ and partial derivative $\partial_{t}$. In (7.2) we recognized any $p$-form $f^{p}$ can be decomposed by $f^{p}=f_{t}^{p}+f_{s}^{p}$, where $f_{t}^{p}$ has a time component and $f_{s}^{p}$ does not. By definition, since $\mathrm{d} t \wedge \mathrm{d} t \equiv 0$ we also have $\mathrm{d} t \wedge \partial_{t} f_{t}^{p} \equiv 0$, and hence, we need to consider only component $\mathrm{d} t \wedge \partial_{t} f_{s}^{p}$. (In (7.4) the operator also acts only on $p$-forms with no time component.)

The finite dimensional wave problem is constructed by mapping differential forms to cochains. Consequently, the finite dimensional counterpart of $\mathrm{d} t \wedge \partial_{t} f_{s}^{p}$ is $\mathcal{C}_{t} \partial_{t} \mathcal{C}_{s} f_{s}^{p} \mathrm{~d} t$. When this acts on pair $\left(\tau^{1}, \sigma^{p}\right) \in T_{t}^{1} \times T_{s}^{p}$ $-i . e$. , on a pair consisting of a 1-cell in time and a spatial $p$-cell- one gets

$$
\mathcal{C}_{t} \partial_{t} \mathcal{C}_{s} f_{s}^{p} \mathrm{~d} t\left(\tau^{1}, \sigma^{p}\right)=\int_{\tau^{1}} \partial_{t} \mathcal{C}_{s} f_{s}^{p}\left(\sigma^{p}\right) \mathrm{d} t,
$$

where $\mathcal{C}_{s} f_{s}^{p}\left(\sigma^{p}\right) \in \mathbb{R}$. Accordingly, $\int_{\tau^{1}} \partial_{t} \mathcal{C}_{s} f_{s}^{p}\left(\sigma^{p}\right) \mathrm{d} t$ is of the form $\int_{\tau^{1}} \partial_{t} \beta \mathrm{d} t$ for some time-dependent scalar function $\beta$.

As chains are formal sums of cells, when $\mathcal{C}_{t} \partial_{t} \mathcal{C}_{s} f_{s}^{p} \mathrm{~d} t$ acts on a pair of chains $\left(c^{1}, c^{p}\right) \in C_{t}^{1} \times C_{s}^{p}$, the result is

$$
\mathcal{C}_{t} \partial_{t} \mathcal{C}_{s} f_{s}^{p} \mathrm{~d} t\left(c^{1}, c^{p}\right)=\sum_{j \in I_{t}} \sum_{i \in I_{s}} \alpha_{j} \alpha_{i} \int_{\tau_{j}^{1}} \partial_{t} \mathcal{C}_{s} f_{s}^{p}\left(\sigma_{i}^{p}\right) \mathrm{d} t
$$


where $I_{t}$ are $I_{s}$ are the index sets of 1-cells in time complex and $p$-cells in spatial complex, respectively.

Summing up, the finite dimensional counterpart of $\mathrm{d} t \wedge \partial_{t}$ is $\mathcal{C}_{t} \partial_{t} \cdot \mathrm{d} t$, and when this acts on 1-chain $c^{1}$ of the time complex one gets

$$
\mathcal{C}_{t} \partial_{t} \cdot \mathrm{d} t\left(c^{1}\right)=\sum_{j \in I_{t}} \alpha_{j} \int_{\tau_{j}^{1}} \partial_{t} \cdot \mathrm{d} t
$$

(4) The finite dimensional counterpart of $\star \partial_{t} \star$ is $*^{-1} \partial_{t} *$.

(5) Operator $\mathrm{d} t \wedge \mathrm{d}$ is akin to $\mathrm{d} t \wedge \partial_{t}$. The partial derivative in time is just replaced with exterior derivative. Consequently, its finite dimensional counterpart is $\mathcal{C}_{t} \mathrm{~d} \cdot \mathrm{d} t$, and when this acts on 1-chain $c^{1}$ of the time complex, the result is

$$
\mathcal{C}_{t} \mathrm{~d} \cdot \mathrm{d} t\left(c^{1}\right)=\sum_{j \in I_{t}} \alpha_{j} \int_{\tau_{j}^{1}} \mathrm{~d} \cdot \mathrm{d} t .
$$

(6) Operator $\mathrm{d} t \wedge \star \mathrm{d} \star= \pm \mathrm{d} t \wedge \delta$ maps to $\mathcal{C}_{t} *^{-1} \mathrm{~d} * \cdot \mathrm{d} t$, when this acts on 1-chain $c^{1}$ of the time complex, one gets

$$
\mathcal{C}_{t} *^{-1} \mathrm{~d} * \cdot \mathrm{d} t\left(c^{1}\right)=\sum_{j \in I_{t}} \alpha_{j} \int_{\tau_{j}^{1}} *^{-1} \mathrm{~d} * \cdot \mathrm{d} t
$$

Summing up, the images of the operators are

\begin{tabular}{lllllll}
\hline Operator & $\mathrm{d}$ & $\star \mathrm{d} \star$ & $\mathrm{d} t \wedge \partial_{t}$ & $\star \partial_{t} \star$ & $\mathrm{d} t \wedge \mathrm{d}$ & $\mathrm{d} t \wedge \star \mathrm{d} \star$ \\
\hline Image & $\mathrm{d}$ & $*^{-1} \mathrm{~d} *$ & $\mathcal{C}_{t} \partial_{t} \cdot \mathrm{d} t$ & $*^{-1} \partial_{t} *$ & $\mathcal{C}_{t} \mathrm{~d} \cdot \mathrm{d} t$ & $\mathcal{C}_{t} *^{-1} \mathrm{~d} * \cdot \mathrm{d} t$ \\
\hline
\end{tabular}

To simplify the notation and to make it easier to read, from now on we will denote by upper case cochains that correspond to spatial differential forms (expressed in the lower case), such as $F=\mathcal{C}_{s} f$. The de Rham operator $\mathcal{C}_{t}$ is still, however, made explicit.

The $p$-forms involved in (7.4), $p=0, \ldots, n$, are mapped to finite dimensional spaces as follows:

\begin{tabular}{lllll}
\hline Differential form & $f_{s}^{p}$ & $f_{*}^{p}$ & $g_{s}^{p}$ & $\mathrm{~d} t \wedge g_{*}^{p}$ \\
\hline Cochain & $F_{s}^{p}$ & $F_{*}^{p}$ & $G_{s}^{p}$ & $\mathcal{C}_{t} G_{*}^{p} \mathrm{~d} t$ \\
\hline
\end{tabular}

Substituting the finite dimensional counterparts of the operators and the differential forms back to (7.4) results in a system of equations which is the finite dimensional wave problem

Definition 8.13. The finite dimensional wave problem is

$$
D \underline{f}=\underline{g},
$$


where

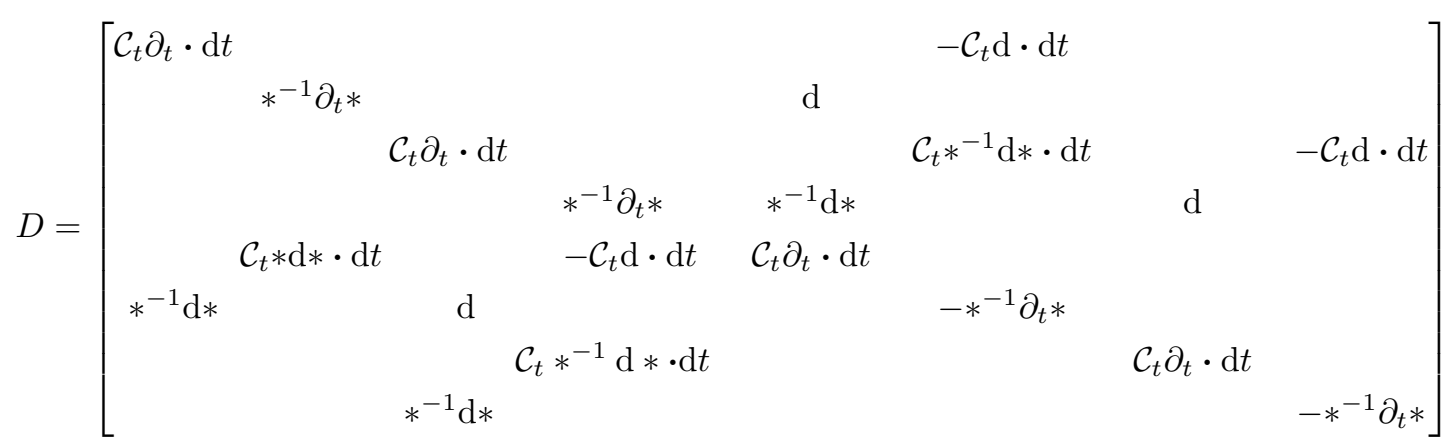

and

$$
\begin{aligned}
\underline{f} & =\left[F_{s}^{3}, F_{*}^{3}, F_{s}^{1}, F_{*}^{1}, F_{s}^{2}, F_{*}^{2}, F^{0}, F_{*}^{0}\right]^{T}, \\
\underline{g} & =\left[\mathcal{C}_{t} G_{*}^{3} \mathrm{~d} t, G_{s}^{3}, \mathcal{C}_{t} G_{*}^{1} \mathrm{~d} t, G_{s}^{1}, \mathcal{C}_{t} G_{*}^{2} \mathrm{~d} t, G_{s}^{2}, \mathcal{C}_{t} G_{*}^{0} \mathrm{~d} t, G^{0}\right]^{T} .
\end{aligned}
$$

\subsection{Finite dimensional electromagnetic wave problem}

To exemplify the use of the finite dimensional wave problem, let us next instantiate the finite dimensional electromagnetic wave problem. Again, to simplify the notation we write $\varepsilon$ for $*_{\epsilon}$ and $\nu$ for $*_{\nu}$. The finite dimensional counterpart of the Maxwell equations (7.5) - (7.8) is

$$
\begin{aligned}
\mathrm{d} B & =0 \\
\mathcal{C}_{t} \mathrm{~d} E \mathrm{~d} t+\mathcal{C}_{t} \partial_{t} B \mathrm{~d} t & =0 \\
-*^{-1} \partial_{t} \varepsilon E+*^{-1} \mathrm{~d} \nu B & =J_{\star} \\
-\mathcal{C}_{t} *^{-1} \mathrm{~d} \varepsilon E \mathrm{~d} t & =-\mathcal{C}_{t} Q_{\star} \mathrm{d} t
\end{aligned}
$$

where $J_{\star}=\mathcal{C}_{s} \star j$ and $Q_{\star}=\mathcal{C}_{s} \star q$.

Next, we assume that (8.6) and (8.9) hold as initial conditions. Then equations (8.7) and (8.8) over the chains of complexes $T_{t}$ and $T_{s}$ specify a finite dimensional approximation of an electromagnetic wave in $\Omega_{t} \times \Omega_{s}$ as explained next.

We start from (8.7) and term $\mathcal{C}_{t} \mathrm{~d} E \mathrm{~d} t$. At first, we recognize that $\mathrm{d} t=\star_{t} 1$, i.e., relation (7.3), and (8.4) imply

$$
\mathcal{C}_{t} \mathrm{~d} t\left(\tau^{1}\right)=\mathcal{C}_{t} \star 1\left(\tau^{1}\right)=\mathcal{C}_{t} 1\left(H \tau^{1}\right)=\frac{\left|\tau^{1}\right|}{\left|\tilde{\tau}^{0}\right|} \mathcal{C}_{t} 1\left(\tilde{\tau}^{0}\right)=\left|\tau^{1}\right| \mathcal{C}_{t} 1\left(\tilde{\tau}^{0}\right),
$$

where 1-cell $\tau^{1} \in T_{t}$ and 0-cell $\tilde{\tau}^{0} \in \tilde{T}_{t}$ is the dual pair of $\tau^{1}$. Consequently, we have

$$
\mathcal{C}_{t} \mathrm{~d} E \mathrm{~d} t\left(\tau^{1}\right)=\left|\tau^{1}\right| \mathcal{C}_{t} \mathrm{~d} E\left(\tilde{\tau}^{0}\right)=\left|\tau^{1}\right| \int_{\tilde{\tau}^{0}} \mathrm{~d} E=\left|\tau^{1}\right| \mathrm{d} E\left(\tilde{\tau}^{0}\right) .
$$

Informally, $\left|\tau^{1}\right| \mathrm{d} E\left(\tilde{\tau}^{0}\right)$ is $\mathrm{d} E$ at moment $t=\tilde{\tau}^{0}$ multiplied by length of the "time step" $\left|\tau^{1}\right|$.

The Stokes theorem implies the second term of (8.7) satisfies

$$
\mathcal{C}_{t} \partial_{t} B \mathrm{~d} t\left(\tau^{1}\right)=\int_{\tau^{1}} \partial_{t} B \mathrm{~d} t=\int_{\partial \tau^{1}} B=B\left(\tau_{e}^{0}\right)-B\left(\tau_{s}^{0}\right),
$$

where $\partial \tau^{1}=\tau_{e}^{0}-\tau_{s}^{0}$ is the boundary of the time step $\tau^{1}$. That is, $\tau_{e}^{0}$ is the "end node" and $\tau_{s}^{0}$ is the "start node" of $\tau^{1}$. 
Substituting (8.10) and (8.11)-(8.7) results in

$$
B\left(\tau_{e}^{0}\right)=B\left(\tau_{s}^{0}\right)-\left|\tau^{1}\right| \mathrm{d} E\left(\tilde{\tau}^{0}\right) .
$$

Since $\partial \tau^{1}=\tau_{e}^{0}-\tau_{s}^{0}$ and $H \tau^{1}=\left|\tau^{1}\right| \tilde{\tau}^{0}$, the notation can be shortened by writing

$$
B\left(\partial \tau^{1}\right)=-\mathrm{d} E\left(H \tau^{1}\right) .
$$

The second equation of the finite dimensional electromagnetic wave model is (8.8). According to (7.3) $1=\star_{t} \mathrm{~d} t$, and hence, (8.4) implies

$$
1\left(\tau^{0}\right)=\star_{t} \mathrm{~d} t\left(\tau^{0}\right)=\mathcal{C}_{t} \mathrm{~d} t\left(H \tau^{0}\right)=\frac{1}{\left|\tilde{\tau}^{1}\right|} \mathcal{C}_{t} \mathrm{~d} t\left(\tilde{\tau}^{1}\right),
$$

where $\tilde{\tau}^{1} \in \tilde{T}_{t}$ is the dual counterpart of $\tau^{0} \in T_{t}$. Consequently, the first term of (8.8) can be given by

$$
-*^{-1} \partial_{t} \varepsilon E\left(\tau^{0}\right)=-\frac{1}{\left|\tilde{\tau}^{1}\right|} \mathcal{C}_{t^{*}}{ }^{-1} \partial_{t} \varepsilon E \mathrm{~d} t\left(\tilde{\tau}^{1}\right)=-\frac{1}{\left|\tilde{\tau}^{1}\right|} *^{-1} \int_{\tilde{\tau}^{1}} \partial_{t} \varepsilon E \mathrm{~d} t .
$$

Thanks to the Stokes theorem we also have

$$
\int_{\tilde{\tau}^{1}} \partial_{t} \varepsilon E \mathrm{~d} t=\int_{\partial \tilde{\tau}^{1}} \varepsilon E=\varepsilon\left(E\left(\tilde{\tau}_{e}^{0}\right)-E\left(\tilde{\tau}_{s}^{0}\right)\right),
$$

where $\partial \tilde{\tau}^{1}=\tilde{\tau}_{e}^{0}-\tilde{\tau}_{s}^{0}$. Hence, we have

$$
-*^{-1} \partial_{t} \varepsilon E\left(\tau^{0}\right)=\frac{1}{\left|\tilde{\tau}^{1}\right|} *^{-1} \varepsilon\left(E\left(\tilde{\tau}_{s}^{0}\right)-E\left(\tilde{\tau}_{e}^{0}\right)\right) .
$$

Subsituting this back to (8.8) yields

$$
*^{-1} \varepsilon E\left(\tilde{\tau}_{e}^{0}\right)=*^{-1} \varepsilon E\left(\tilde{\tau}_{s}^{0}\right)+\left|\tilde{\tau}^{1}\right|\left(*^{-1} \mathrm{~d} \nu B\left(\tau^{0}\right)-J_{\star}\left(\tau^{0}\right)\right) .
$$

Since we have $\partial \tilde{\tau}^{1}=\tilde{\tau}_{e}^{0}-\tilde{\tau}_{s}^{0}, H \tau^{0}=\frac{1}{\left|\tilde{\tau}^{1}\right|} \tilde{\tau}^{1}$, and $* *^{-1}=1$, eq. (8.13) can be shortened and be given by

$$
\varepsilon E\left(\partial H \tau^{0}\right)=\mathrm{d} \nu B\left(\tau^{0}\right)-* J_{\star}\left(\tau^{0}\right) .
$$

Employing (8.12) and (8.14) the finite dimensional electromagnetic wave problem generalizing Yee's original approach can now be given by

Definition 8.14. The finite dimensional electromagnetic wave problem is: for given current $J_{\star}$, permittivity $\varepsilon$, reluctivity $\nu\left(=\mu^{-1}\right.$, where $\mu$ is permeability), and initial and boundary conditions, find a bilinear $E$ : $\tilde{C}_{t}^{0} \times C_{s}^{1} \rightarrow \mathbb{R}$ and $B: C_{t}^{0} \times C_{s}^{2} \rightarrow \mathbb{R}$ such that

$$
\begin{aligned}
B\left(\partial c_{t}^{1}, c_{s}^{2}\right) & =-\mathrm{d} E\left(H c_{t}^{1}, c_{s}^{2}\right) & & \forall c_{t}^{1} \in C_{t}^{1}, c_{s}^{2} \in C_{s}^{2}, \\
\varepsilon E\left(\partial H c_{t}^{0}, \tilde{c}_{s}^{2}\right) & =\mathrm{d} \nu B\left(c_{t}^{0}, \tilde{c}_{s}^{2}\right)-* J_{\star}\left(c_{t}^{0}, \tilde{c}_{s}^{2}\right) & & \forall c_{t}^{0} \in C_{t}^{0}, \tilde{c}_{s}^{2} \in \tilde{C}_{s}^{2} .
\end{aligned}
$$

hold.

Remark 8.15. The original Yee scheme is a special case of (8.15) and (8.16), and the equations can be solved in the same fashion with the "leap-frog" method.

Remark 8.16. The problem is driven (with the boundary conditions or) with 1-cochain $J_{\star}$, that corresponds to currents on 1-cells. Some electromagnetic problems are driven with a "source voltage" $e_{s}$. In this case one writes $E_{s}$ in place of $J_{\star}$. If the domain contains conductive materials, then in place of $* J_{\star}$ one writes $\sigma E+* J_{\star}$, where $\sigma=*_{\sigma}$ is conductivity. 
Remark 8.17. To solve for cochains $E$ and $B$ (and for $\varepsilon E$ and $\nu B$ ) from (8.15) and (8.16) one does not need any kind of finite element basis functions. For this, notice first that fields and cochains are concepts dual to each other to express the same information. By definition, def. 8.3, given a field, such as $e$ or $b$, one can always find the values of cochains $E$ and $B$, respectively, on any chain by integration. The other way around, given a cochain, the corresponding field is found with a limit value process. Consequently, given a field or a cochain, from this one can always find the other.

Next, equations. (8.15) and (8.16) are network equations, where $\varepsilon=* \epsilon$ and $\nu=* \nu$ are about impedances. And now, as in case of circuit theory one does not need any basis functions to solve for (the cochains) branch voltages and circuit currents, also $E$ and $B$ can be solved explicitly without any interpolation to the interiors of the cells.

Compare, the very idea of the finite element method is to set one of the differential equations exactly and the other in the weak sense. To impose differential equations in the weak sense, one needs constitutive laws at all ordinary points of space, and for this, the finite element basis functions are a necessity to interpolate fields within the elements from the degrees of freedom. As we have already noticed earlier, Yee-like schemes impose both differential equations exactly, whereas the constitutive laws only at the points where the $p$-cells of the primal complex intersect with the $(n-p)$-cells. For this reason setting up the system of equations does not involve any interpolation.

Remark 8.18. Some have surmised the network interpretation implied there existed a finite dimensional version of electromagnetic theory leaving the notion of fields and differential equations unnecessary. While eqs. (8.15) and (8.16) can indeed be interpreted as network equations, and $\varepsilon=* \epsilon$ and $\nu=* \nu$ as impedances, the specification of these impedances depend on $\star_{\epsilon}$ and $\star_{\mu}$ and on the material parameters (that are specified with respect to some metric). This is to say, without the local Hodge mappings we would not have material parameters and would not be able to predict the impedances required to connect the Faraday law to the Ampère law.

\section{Higher ORDER APPROXIMATIONS OF THE WAVE PROBLEM}

The solutions of the finite dimensional wave problem are the real numbers cochains yield on chains. Typically, these numbers alone are not enough, and in addition, one wants to view the fields within the cells. For this a converse to the de Rham map is needed, and this is he Whitney map.

\subsection{Whitney map and lowest order Whitney forms}

From now on, we assume triangulation $T$ is a regular simplicial complex and denote it by $S$. To shorten the notation, the $p$-cochain whose value is one at simplex $\sigma^{p}$ and zero at all other $p$-simplices is also denoted by $\sigma^{p}$. Such cochains form a basis for $C_{p}$.

Definition 9.1. A linear map from $p$-cochains to $p$-forms in a complex $S, p=0, \ldots, n$, is the (lowest order) Whitney map $\mathcal{W}$, if

(1) for $p=0,0$-cochain $x$ maps to the barycentric function $\lambda_{x}$, and

(2) for $1 \leq p \leq n, p$-cochain $x_{0} x_{1} \ldots x_{p}$ corresponding to $p$-simplex $x_{0} x_{1} \ldots x_{p} \in S$ maps to

$$
\mathcal{W}\left(x_{0} x_{1} \ldots x_{p}\right)=p ! \sum_{i=0}^{p}(-1)^{i} \lambda_{i} \mathrm{~d} \lambda_{0} \wedge \ldots \widehat{\mathrm{d}}_{i} \ldots \wedge \mathrm{d} \lambda_{p},
$$

where the hat in $\widehat{\mathrm{d}}_{i}$ means that the term is omitted from the product [39, VII, 11.16].

Map $\mathcal{W}$ is straightforwardly extended to all cochains by linearity. 
Definition 9.2. The lowest order Whitney space is $W^{p}=\operatorname{span}\left\{\mathcal{W}\left(\sigma^{p}\right) \mid \sigma^{p} \in S\right\}$, and the elements of $W^{p}$ are Whitney $p$-forms.

Remark 9.3. Whitney forms are "differential forms in a complex" as described in [39, p. 226]. This means that on the inter-element boundaries only the tangential trace of the differential form exists. Accordingly, (9.1) is meaningful even though the barycentric functions are not smooth. For an example, in dimension $n=3$, the Whitney 1-form, also known as the "edge element", is $\mathcal{W}\left(x_{0} x_{1}\right)=\lambda_{0} \mathrm{~d} \lambda_{1}-\lambda_{1} \mathrm{~d} \lambda_{0}$.

Remark 9.4. The defining properties of the Whitney map are [39, VII, 11]:

(i) If $\sigma$ is a simplex of $S$, then $\mathcal{W}(\sigma)=0$ in $\Omega_{s}-\operatorname{St}(\sigma)$,

(ii) $\mathcal{W} \mathrm{d}=\mathrm{d} \mathcal{W}$,

(iii) $\mathcal{C W}=$ id, and

(iv) $\mathcal{W} I^{0}=1$,

where $\operatorname{St}(\sigma)$ denotes the star of simplex $\sigma, I^{0}$ is the unit 0 -cochain of $S$, and 1 is the real function equal to one in $\Omega_{s}$. At will, one may first adopt (i)-(iv) as the definition of the Whitney map, and then instantiate the lowest order Whitney forms (9.1) from this.

\subsection{Strategy for higher order approximations}

Next, we introduce higher order approximations of the generalized wave problem. For this we extend the finite dimensional wave problem to exploit higher order Whitney forms.

The key strategic issue behind the extension is, the solution of the finite dimensional wave problem does not require any finite element kind of basis functions. These are needed only for interpolation.

The solutions of the finite dimensional problem fulfil the differential equations exactly in the sense that operators $\mathrm{d}$ and $\mathcal{C}$ commute, Corollary 8.5, whereas the constitutive laws become approximated at a finite amount of points as explained in Remark 8.12. Furthermore, since the Hodge-relation is a local linear map between tangent spaces of each point, Remark 4.2, the higher order finite dimensional approaches should just increase the dimension of the underlying functional spaces, add the number of points at which the constitutive laws are approximated, and preserve the local character of constitutive laws. This implies, it is justified to employ higher order approximations without changing the actual solution process at all.

Remark 9.5. In other words, as the Hodge operator is a local map between the tangent spaces at each point in space and time, neither the tangent spaces of the neighbouring points nor higher order approximations have any effect to the Hodge operator. Accordingly, the usage of higher order elements do not affect the constitutive laws. In addition, as explained in Remark 8.17, Yee-like schemes convert the differential equations to network equations. As "higher order network equations" made very little sense, the usage of higher order elements either affect the differential equations. Consequently, the higher order approximation boils down to the post processing stage and to the interpolation of cochains within the cells. This applies both to space and time, and this is why the explicit recognition of the Hodge operator also in time, equation (7.3), is important.

We will introduce higher order approximations to the finite dimensional wave problem which rely on a subdivision of the complex and on preserving the explicit solution process. To interpolate cochains in space and time, higher order Whitney forms are employed. Such an approach to solve finite dimensional wave problems is realized in reference [30], and the next Sections 9.3 and 9.4 will follow the approach therein.

\subsection{Subdivision of the complex}

The first stage is to introduce a subdivision strategy of $S$. In this we will employ Rapetti's and Bossavit's idea [32] mapping simplices to their so called homothetic images.

Let us call the set of $l$-tuples of non-negative integers that sum to $k \in \mathbb{N}$ by the name $\mathcal{I}(l, k)$ and an element of $\mathcal{I}(n+1, k)$ by $\mathbf{k}=\left(k_{0}, \ldots, k_{n}\right)$. 


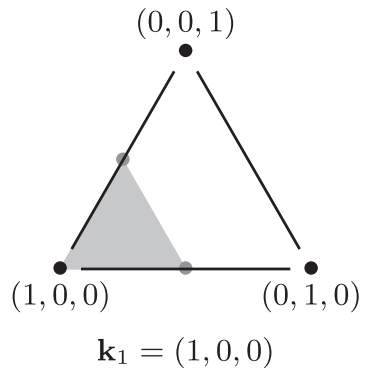

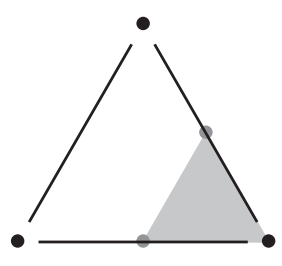

$\mathbf{k}_{2}=(0,1,0)$



$\mathbf{k}_{3}=(0,0,1)$

Figure 2. In dimension $n=2$ and for $k=1$, set $\mathcal{I}(3,1)$ has three elements $\mathbf{k}_{1}=(1,0,0)$, $\mathbf{k}_{2}=(0,1,0)$, and $\mathbf{k}_{3}=(0,0,1)$. The thick lines bound a 2 -simplex $\sigma^{2}$, and the smaller grey triangles show the images $h\left(\sigma^{2}, \mathbf{k}_{1}\right), h\left(\sigma^{2}, \mathbf{k}_{2}\right)$, and $h\left(\sigma^{2}, \mathbf{k}_{3}\right)$.
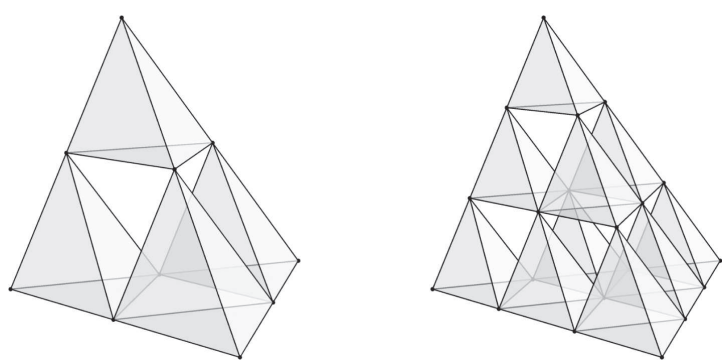

Figure 3. Left: in dimension $n=3$ and for $k=1$, set $\mathcal{I}(4,1)$ has four elements. The corresponding images $h\left(\sigma^{3}, \mathbf{k}\right), \mathbf{k} \in \mathcal{I}(4,1)$ are shown in gray. The cell in the middle shown in white is an octahedron. It corresponds to the union of two pyramids opposite to each other. Right: the same for $k=2$.

We introduce map $h(\cdot, \mathbf{k})$ from (the point set of) $\sigma^{n}$ to itself as follows. Given an $n$-simplex $\sigma^{n}$ by its vertices $x_{0} x_{1} \ldots x_{n}$, and the barycentric coordinates $\lambda(x)=\left(\lambda_{0}(x), \ldots, \lambda_{n}(x)\right)$ of a point $x \in \sigma^{n} \subset \mathbb{R}^{n}$, the value $h(x, \mathbf{k})$ is defined to be the point whose barycentric coordinates are $\frac{1}{(k+1)}\left(\lambda_{0}(x)+k_{0}, \ldots, \lambda_{n}(x)+k_{n}\right)$. In other words,

$$
h: \sigma^{n} \times \mathcal{I}(n+1, k) \rightarrow \sigma^{n},(x, \mathbf{k}) \mapsto \frac{\lambda_{0}(x)+k_{0}}{k+1} x_{0}+\cdots+\frac{\lambda_{n}(x)+k_{n}}{k+1} x_{n} .
$$

Function $h(\cdot, \mathbf{k})$ maps the $n$-simplex $\sigma^{n}$ to a homothetic image of itself, and the same is true for every subsimplex $\sigma^{p}$ of $\sigma^{n}$. (The subsimplices of $n$-simplex $\sigma^{n} \in S^{n}$ are the $p$-simplices of $S$ that are subsets of $\sigma^{n}$.) Some examples are shown in Figures 2 and 3. Following [32] we call the homothetic images of the simplices of $S$ by the name "small simplices". In addition, both a small simplex and its value, i.e., the point set, will be denoted by $h\left(\sigma^{p}, \mathbf{k}\right)$.

Thereafter we can fix $k$ and form a subdivision $T$ of $S$ which contains the small simplices $\left\{h\left(\sigma^{n}, \mathbf{k}\right): \sigma^{n} \in\right.$ $\left.S^{n}, \mathbf{k} \in \mathcal{I}(n+1, k)\right\}$ as cells. We stress out that not all the elements of $T$ are homothetic images of the simplices of $S$. For instance, in Figure 3, right, there are three octahedra and one inverted tetrahedron that are not small simplices. Subdivision $T$ is a refined cell complex.

Once the subdivision $T$ and its dual complex $\tilde{T}$ (fulfilling Def. 8.9) are formed, the finite dimensional wave problem can be solved in the usual manner. We assume now the finite dimensional wave problem is solved with a pair of complexes $(T, \tilde{T})$. The solutions are arrays of reals numbers that correspond to the values cochains yield on the chains of complex T. By definition of the de Rham map, Definition 8.3, these real numbers correspond to integrals of fields over the elements of $T$. 


\subsection{Whitney forms of order $k \in \mathbb{N}$}

The remaining step is to interpolate the cochains. For this we will employ the higher order Whitney forms introduced in [32], following the interpolating strategy of chp. 3 of [30]. The higher order Whitney forms in [32] are defined as products of the barycentric functions and the lowest order Whitney forms.

As explained above, function $h(\cdot, \mathbf{k})$ maps $n$-simplex $\sigma^{n} \in S$ and its subsimplices to their homothetic images. Symbol $h\left(\sigma^{p}, \mathbf{k}\right)$ is employed also to point to the $p$-cochain whose value is one at $h\left(\sigma^{p}, \mathbf{k}\right)$ and zero at other small $p$-simplices of $S$.

At first, we define a map $\mathcal{V}$ from cochains over the small simplices of $S$ to differential forms in $S$, so that the image of $\mathcal{V}$ is the space of $k$ th order Whitney forms.

Definition 9.6. A linear map from $p$-cochains to $p$-forms in $S$ is map $\mathcal{V}$, if the $p$-cochain associated to small $p$-simplex $h\left(\sigma^{p}, \mathbf{k}\right)=h\left(\sigma^{p},\left(k_{0}, \ldots, k_{n}\right)\right)$ of $\sigma^{n}=x_{0} \ldots x_{n}$ maps to

$$
\mathcal{V}\left(h\left(\sigma^{p}, \mathbf{k}\right)\right)=\left(\prod_{i=0}^{n} \lambda_{i}^{k_{i}}\right) \mathcal{W}\left(\sigma^{p}\right),
$$

Again, map $\mathcal{V}$ extends to all cochains by linearity.

Definition 9.7. The $k$ th order Whitney space is

$$
W_{k}^{p}=\operatorname{span}\left\{\mathcal{V}\left(h\left(\sigma^{p}, \mathbf{k}\right)\right) \mid \sigma^{p} \in S, \mathbf{k} \in \mathcal{I}(n+1, k-1)\right\} .
$$

The elements of $W_{k}^{p}$ are called $k$ th order Whitney $p$-forms.

Remark 9.8. The functions $\mathcal{V}\left(h\left(\sigma^{p}, \mathbf{k}\right)\right)$ associated with the small simplices of $S$ are not linearly independent. Consequently, they do not either constitute a basis for $W_{k}^{p}$. In other words, the functions $\mathcal{V}\left(h\left(\sigma^{p}, \mathbf{k}\right)\right)$ are not finite element kind of basis functions.

As stated in [32] these higher order Whitney forms retain some properties of the lowest order Whitney forms. Namely, they satisfy a partition of unity property, and the spaces $W_{k}^{p}, p=0, \ldots, n$, constitute an exact sequence. This justifies calling the $k$ th order forms by the name higher order Whitney forms. However, we point out that the map $\mathcal{V}$ does not share all the properties of the Whitney map $\mathcal{W}$. For this reason, interpolating cochains with these higher order Whitney forms is either not as straightforward as in the lowest order case.

To recognize the issue, recall property (iii) of the Whitney map; $\mathcal{C W}=$ id. When applied to the cochain $\sigma^{p}$, this implies that the integral of $\mathcal{W}\left(\sigma^{p}\right)$ is one over the simplex $\sigma^{p}$ and zero over all other $p$-simplices. Accordingly, in case of the lowest order Whitney's cochain $X$ is interpolated simply as $\mathcal{W}(X)$, and the coefficients $\alpha_{i}$ of the interpolant $\sum_{i} \alpha_{i} \mathcal{W}\left(\sigma_{i}\right)$ are the values of $X$ on the corresponding simplices $\sigma_{i}$.

In case of $\mathcal{V}$, however, it is not true that for all small simplices $v$

$$
\int_{v} \mathcal{V}(v)=1 \quad \text { and } \quad \int_{v^{\prime}} \mathcal{V}(v)=0 \quad \forall v^{\prime} \neq v
$$

As the $k$ th order forms corresponding to small simplices are not linearly independent, the dimension of $W_{k}^{p}$ is lower than the number of small simplices. This implies there exists $p$-cochains $X$ such that $\mathcal{C} w \neq X$ for all $w \in W_{k}^{p}$. In other words, not all cochains $X$ can be given as de Rham images of the $k$ th order forms.

To deal with the linear dependency, we omit some of the small simplices so that the number of small simplices matches the dimension of $W_{k}^{p}$. This amounts to restricting the map $\mathcal{V}$ to a subspace isomorphic to $W_{k}^{p}$. Then we can make property (iii) to hold in this subspace.

In more detail, the coefficients of the interpolant $\sum_{v} \alpha_{v} \mathcal{V}(v)$ are linear combinations of the values of $X$ on the small simplices; $\alpha_{v}$ depends not only on the value of $X$ at $v$, but also on the values of $X$ on other small 
simplices in the $n$-simplex containing $v$. To see how the coefficients are determined, consider $\sigma^{n} \in S$, and form a matrix $\mathbf{V}$, indexed over small simplices of $\sigma^{n}$, such that

$$
\mathbf{V}_{v^{\prime}}^{v}=\int_{v} \mathcal{V}\left(v^{\prime}\right)
$$

With respect to this basis, matrix $\mathbf{V}$ corresponds to the linear map $\mathcal{C} \mathcal{V}$ from the space of cochains (over small simplices of $\sigma^{n}$ ) to itself.

After restricting map $\mathcal{V}$ to a subspace by omitting redundant small simplices, matrix $\mathbf{V}$ becomes invertible. Then, in each $n$-simplex of $S$ and in this subspace property $\mathcal{C} \mathcal{V} \mathbf{V}^{-1}=$ id holds. Consequently, we can interpolate $X$ to Whitney form $\mathcal{V} \mathbf{V}^{-1} X$, and the integrals of the interpolant will match with the values of $X$ on the small simplices that were not omitted.

The coefficient $\alpha_{v}=\sum_{v^{\prime}}\left(\mathbf{V}^{-1}\right)_{v^{\prime}}^{v} X\left(v^{\prime}\right)$ depends on the values of $X$ and is determined by the $v$-row of $\mathbf{V}^{-1}$. It is shown in [30] that the coefficient is well-defined and does not depend on the $n$-simplex considered. If $v$ belongs to multiple $n$-simplices, $\alpha_{v}$ depends only on the values on those small simplices that are contained in all of the $n$-simplices containing $v$.

Summing up, the solutions of finite dimensional wave problems are images of the de Rham map, and these cochains can now be interpolated elementwise with map $\mathcal{V} \mathbf{V}^{-1}$; in simplex $\sigma^{n} \in S^{n}$ the $k$ th order differential form corresponding to $p$-cochain $X$ is

$$
\sum_{v} \alpha_{v} \mathcal{V}(v)=\sum_{v}\left(\sum_{v^{\prime}}\left(\mathbf{V}^{-1}\right)_{v^{\prime}}^{v} X\left(v^{\prime}\right)\right) \mathcal{V}(v),
$$

where the $v$ 's are the small $p$-simplices of $\sigma^{n}$.

Remark 9.9. Be aware, the $k$ th order Whitney forms can be employed to interpolate the solution of the finite dimensional wave problem both in time and space.

\section{NUMERICAL EXPERIMENTS}

In this section we demonstrate the solution of the finite dimensional wave problem using the first (i.e., the lowest) and the second order Whitney forms. The test case is an electromagnetic wave. The boundary value problem is driven harmonically, and the solution is integrated in time until reaching harmonic stability within a reasonable tolerance. The aim is to examine the convergence when the mesh grain tends to zero.

The dimension of the test example is $n=1+3$. We first form a spatial simplicial complex $S_{0}$ exploiting the regular body-centred cubic structure. Thereafter $S_{0}$ is subdivided as explained in Section 9.3. Then, in order to compare the results obtained with the lowest and second order interpolation schemes, the octahedra of $T_{0}$, each surrounded by small tetrahedra as shown in Figure 3, are refined into four tetrahedra. This provides us with a simplicial complex $S_{T 0}$. Once the finite dimensional problem is solved in $S_{T 0}$, the cochains are interpolated to differential forms with the lowest and second order Whitney forms. This makes it possible to compare the effect of the interpolation schemes.

To examine convergence, complex $S_{T 0}$ is refined by considering $S_{T 0}$ first as a simplicial complex $S_{1}$, and then by subdividing $S_{1}$ to $S_{T 1}$ in the same manner as $S_{T 0}$ was generated out of $S_{0}$. This results in an inductive refinement scheme. The finite dimensional wave problem is solved in each complex $S_{T i}, i=0, \ldots, N$ in time until a steady-state situation is achieved with a reasonable tolerance. The solution, i.e., the cochains are interpolated to Whitney forms of order $k=1$ and $k=2$, and then the $L^{2}$-norm is computed and exploited to examine convergence.

The test case is a solution of the finite dimensional electromagnetic wave problem, see Definition 8.14. The units are chosen in a relative manner such that the product of the speed of light $c$ and the unit of time $[t]$ match with the unit of length $[1], c[t]=[l]$. Accordingly, the unit of length is the distance light travels in one unit of time, and the unit of time is the time light takes to propagate the unit of length. 


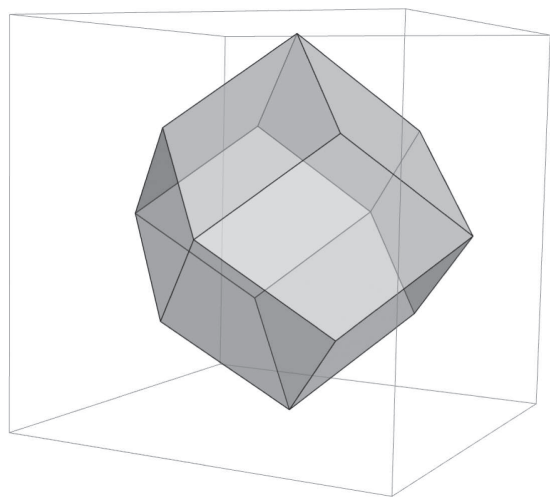

Figure 4. Rhombic dodecahedron.

TABLE 1. $L^{2}$ norm of the difference in the interpolated solutions on consecutive meshes.

\begin{tabular}{|c|c|c|c|c|}
\hline \multicolumn{5}{|c|}{$L^{2}$ norm of the difference $e^{2 j}-e^{j}$} \\
\hline$j$ & 4 & 8 & 16 & 32 \\
\hline Lowest order Whitney 1-forms, $k=1$ & 2.09902 & 0.84525 & 0.39412 & 0.19369 \\
\hline Second order Whitney 1 -forms, $k=2$ & 1.91274 & 0.48106 & 0.12334 & 0.032873 \\
\hline \multicolumn{5}{|c|}{$L^{2}$ norm of the difference $b^{2 j}-b^{j}$} \\
\hline$j$ & 4 & 8 & 16 & 32 \\
\hline Lowest order Whitney 2 -forms, $k=1$ & 3.67633 & 1.48184 & 0.66862 & 0.31981 \\
\hline Second order Whitney 2 -forms, $k=2$ & 3.58869 & 1.14689 & 0.41462 & 0.17195 \\
\hline
\end{tabular}

The domain is a rhombic dodecahedron. Its vertices $( \pm 1,0,0)^{T},(0, \pm 1,0)^{T}$, and $(0,0, \pm 1)^{T}$ are on the boundary of a unit ball, see Figure 4 . The values of relative permittivity, relative reluctivity, and relative conductivity are set to $\epsilon=2.0, \nu=1.0$, and $\sigma=1.0$ with respect to the relative units, respectively.

The boundary condition imposes the value of $E=\mathcal{C} e$ on the boundary edges of the rhombic dodecahedron, where function $e$ is given by

$$
e(x, y, z, t)=\cos (\omega t-k x) \mathrm{d} y+\sin (\omega t-k x) \mathrm{d} z,
$$

wave number $k=2 \pi$, and angular velocity is set to $\omega=2 \pi$ radians per the unit of time. This corresponds to a circular polarized wave propagating in the $x$-direction, whose wavelength is one unit.

The initial triangulation $S_{0}$ was refined to create triangulation $S_{T 0}$ that included 192 tetrahedra. The mesh grain of $S_{T 0}$ is denoted by $j^{-1}=2^{-1}$. Triangulation $S_{T 0}$ was then refined five times to obtain triangulations $S_{T i}, i=1, \ldots, 5$. These triangulations correspond with mesh grains $j^{-1}$, where $j=4,8,16,32,64$. The finite dimensional electromagnetic test problem was solved with these triangulations. Thereafter the cochains were interpolated to Whitney forms of order $k=1$ and $k=2$. The results are shown in Table 1 and in Figure 5 .

The results suggests second order Whitney forms yield a faster convergence as expected. However, magnetic flux density $b$ converges slower than electric field $e$. This is explained by the fact that $b$ is derived from $e$, and that differentiation decreases the degree.

\section{Conclusions}

In this paper we have presented a systematic approach to construct a generalized wave propagation problem that corresponds to a class of wave problems covering at large physics field theories. Then, by introducing 


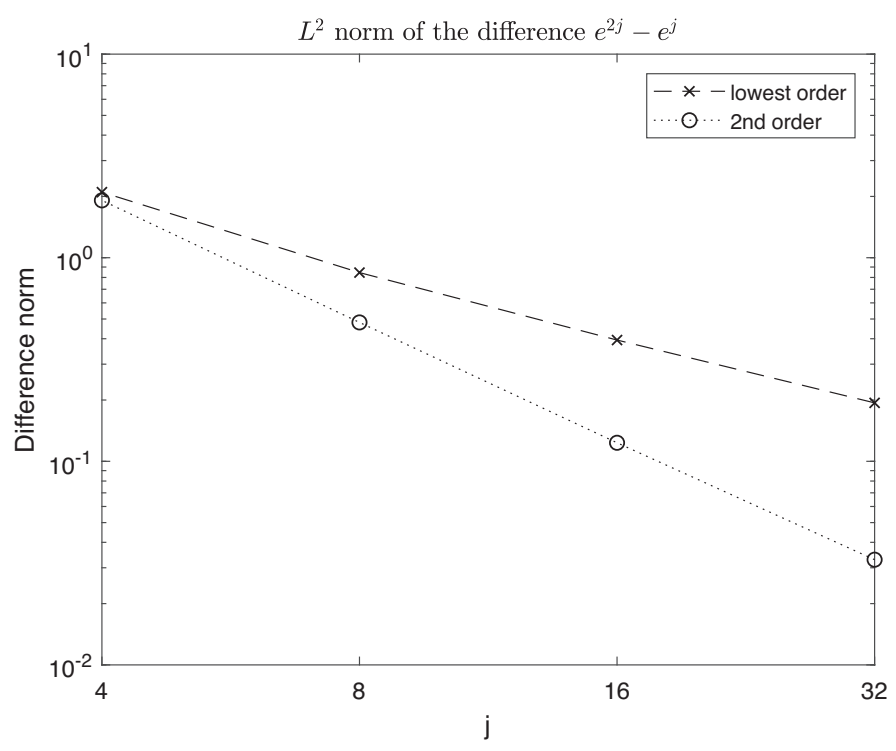

Figure 5. Graph of the results shown in Table 1.

a functor that maps the field notions of the generalized problem to finite dimensional cochain spaces and the (covariant) exterior derivative and the Hodge operator to linear operators between the cochain spaces, a finite dimensional counterpart of the generalized wave propagation problem is obtained. Thereafter it is straightforward to instantiate finite dimensional models for the physics field theories and to construct boundary value problems that can be solved with finite difference kind of schemes.

In the finite dimensional models the counterpart of the exterior derivative is the coboundary operator. This makes it possible express differential equations exactly in finite dimensional spaces. In other words, the exterior derivative, the coboundary operator, and the de Rham map form a commutative diagram. There is no corresponding commutative diagram available for the Hodge operator and the "discrete Hodge operator", and consequently, the constitutive laws cannot be imposed exactly in finite dimensional space. However, one may interpret the constitutive relations are imposed at the finite amount of points where the $p$-cells of the primal triangulation intersect with the $(n-p)$-cells (for the corresponding $p$ and $(n-p)$-vectors). Such a view reveals higher order finite difference schemes can be constructed without changing the actual (explicit) solution process at all. Accordingly, higher order finite difference schemes can be constructed by increasing the dimension of the finite dimensional spaces by subdivision. This then adds also the number of points where the constitutive relations becomes imposed.

To interpret the results, in the postprocessing stage one typically wants to interpolate the cochains back to finite dimensional spaces of differential forms in a complex. For this one can employ Whitney forms of the order $k \in \mathbb{N}$ both in space and time. The test example from electromagnetism demonstrates their usage results in a faster convergence.

Acknowledgements. We would like to acknowledge A. Bossavit, J. Parkkonen, S. Suuriniemi, and T. Tarhasaari for the discussions on the topics of this paper.

\section{REFERENCES}

[1] R. Abraham and J.E. Marsden, Foundations of Mechanics, 2nd edition. Addison-Wesley (1987).

[2] J. Baez and J.P. Muniain, Gauge Fields, Knots and Gravity. Series on Knots and Everything. World Scientific (1994). 
[3] D. Bleecker, Gauge Theories and Variational Principles. Addison-Wesley (1981).

[4] A. Bossavit, How weak is the 'weak solution' in finite element methods? IEEE Trans. Magn. 34 (1998) $2429-2432$.

[5] A. Bossavit, On the geometry of electromagnetism, 1. Affine space. J. Jpn. Soc. Appl. Electromag. Mech. 6 (1998) 17-28.

[6] A. Bossavit, On the geometry of electromagnetism, 2. Geometrical objects. J. Jpn. Soc. Appl. Electromag. Mech. 6 (1998) $114-123$.

[7] A. Bossavit, On the geometry of electromagnetism, 3. Integration, stokes, faraday's law. J. Jpn. Soc. Appl. Electromag. Mech. 6 (1998) 223-240.

[8] A. Bossavit, On the geometry of electromagnetism, 4. Maxwell's house. J. Jpn. Soc. Appl. Electromag. Mech. 6 (1998) $318-326$.

[9] A. Bossavit, Computational electromagnetism and geometry: building a finite-dimensional "Maxwell's house". J. Jpn. Soc. Appl. Electromag. Mech. 7 (1999) 150-159.

[10] A. Bossavit, Computational electromagnetism and geometry: convergence. J. Jpn. Soc. Appl. Electromag. Mech. 7 (1999) 401-408.

[11] A. Bossavit, Computational electromagnetism and geometry: network constitutive laws. J. Jpn. Soc. Appl. Electromag. Mech. 7 (1999) 294-301.

[12] A. Bossavit, Computational electromagnetism and geometry: from degrees of freedom to fields. J. Jpn. Soc. Appl. Electromag. Mech. 8 (2000) 102-109.

[13] A. Bossavit, Computational electromagnetism and geometry: some questions and answers. J. Jpn. Soc. Appl. Electromag. Mech. 8 (2000) 372-377.

[14] A. Bossavit, Computational electromagnetism and geometry: the "Galerkin hodge". J. Jpn. Soc. Appl. Electromag. Mech. 8 (2000) 203-209.

[15] A. Bossavit, 'Generalized finite differences' in computational electromagnetics. edited by F.L. Teixeira. In: Progress in Electromagnetics Research, PIER, EMW, Cambridge, MA (2001) 45-64.

[16] A. Bossavit, On the notion of anisotropy of constitutive laws: some implications of the 'Hodge implies metric' result. Compel 20 (2001) 233-239.

[17] A. Bossavit and L. Kettunen, Yee-like schemes on a tetrahedral mesh, with diagonal lumping. Int. J. Numer. Model. Electron. Networks Devices Fields, 12 (1999) 129-142.

[18] A. Bossavit and L. Kettunen, Correction to 'Yee-like schemes on staggered cellular grids: a synthesis between FIT and FEM approaches'. IEEE Trans. Magn. 36 (2000) 4050.

[19] A. Bossavit and L. Kettunen, Yee-like schemes on staggered cellular grids: a synthesis between FIT and FEM approaches. IEEE Trans. Magn. 36 (2000) 861-867.

[20] L. Codecasa and M. Politi, Explicit, consistent, and conditionally stable extension of fd-td to tetrahedral grids by fit. IEEE Trans. Magn. 44 (2008) 1258-1261.

[21] H. Flanders, Differential Forms with Application to the Physical Sciences. Dover (1989).

[22] T. Frankel, The Geometry of Physics, an Introduction. 3rd edition, Cambridge Univ. Press, Cambridge, USA (2012).

[23] A. Frölicher and A. Nijenhuis, Theory of vector-valued differential forms: part I. Derivations in the graded ring of differential forms. Indagationes Mathematicae (Proceedings), 59 (1956) 338-350.

[24] A.N. Hirani, Discrete exterior calculus. PhD thesis, Caltech, Pasadena, California, 5 (2003).

[25] W.V.D. Hodge, The Theory and Applications of Harmonic Integrals. Cambridge Univ. Press, Cambridge, USA (1941).

[26] E. Kanso, M. Arroyo, Y. Tong, A. Yavari, J.E. Marsden and M. Desbrun, On the geometric character of stress in continuum mechanics. Z. Angew. Math. Phys. 58 (2007) 1-14.

[27] J. Keäränen, E. Koljonen, T. Tarhasaari and L.Kettunen, Effect of cell type on convergence of wave propagation schemes. IEEE Trans. Magn. 40 (2004) 1452-1455.

[28] L. Kettunen, S. Mönkölä, J. Parkkonen and T. Rossi, General conservation law for a class of physics field theories. arXiv: 1908.10634.

[29] T. Kovanen, T. Tarhasaari and L Kettunen, Formulation of small-strain magneto-elastic problems. https://arxiv.org/abs/ 1602.04966 .

[30] J. Lohi, Discrete exterior calculus and higher order Whitney forms. Master's thesis, University of Jyväskylä (2019).

[31] J. Räbinä, L. Kettunen, S. Mönkölä, and T. Rossi, Generalized wave propagation problems and discrete exterior calculus. ESAIM : M2AN $\mathbf{5 2}(2018)$ 1195-1218.

[32] F. Rapetti and A. Bossavit, Whitney forms of higher degree. SIAM J. Numer. Anal. 47 (2009) 2369-2386.

[33] R. Segev and G. Rodnay, Cauchy's theorem on manifolds. J. Elasticity 56 (1999) 129-144.

[34] T. Tarhasaari, L. Kettunen and A. Bossavit, Some realizations of a discrete Hodge operator: a reinterpretation of finite element techniques. IEEE Trans. Magn. 35 (1999) 1494-1497.

[35] F. Teixeira and W.C. Chew, Lattice electromagnetic theory from a topological viewpoint. J. Math. Phys. 40 (1999) $169-187$.

[36] E. Tonti, A direct discrete formulation of field laws: the Cell method. CMES Comput. Model. Eng. Sci. 2 (2001) $237-258$.

[37] E. Tonti, The Mathematical Structure of Classical and Relativistic Physics. Birkhäuser (2013).

[38] T. Weiland, Time domain electromagnetic field computation with finite difference methods. Int. J. Numer. Model. Electron. Networks Devices Fields 9 (1996) 295-319.

[39] H. Whitney, Geometric Integration Theory, Princeton Univ. Press, USA (1957).

[40] A. Yavari, On geometric discretization of elasticity. J. Math. Phys. 49 (2008).

[41] K. Yee, Numerical solution of initial boundary value problems involving Maxwell's equations in isotropic media. IEEE Trans. Antennas Propag. 14 (1966) 302-307.

[42] K. Yosida, Functional Analysis, Springer-Verlag, Berlin Heidelberg (1995). 


\section{Subscribe to Open (S2O) A fair and sustainable open access model}

This journal is currently published in open access with no charge for authors under a Subscribe-to-Open model (S2O). Open access is the free, immediate, online availability of research articles combined with the rights to use these articles fully in the digital environment.

$\mathrm{S} 2 \mathrm{O}$ is one of the transformative models that aim to move subscription journals to open access. Every year, as long as the minimum amount of subscriptions necessary to sustain the publication of the journal is attained, the content for the year is published in open access.

\section{Ask your library to support open access by subscribing to this $\mathrm{S} 2 \mathrm{O}$ journal.}

Please help to maintain this journal in open access! Encourage your library to subscribe or verify its subscription by contacting subscribers@edpsciences.org

We are thankful to our subscribers and sponsors for making it possible to publish the journal in open access, free of charge for authors. More information and list of sponsors: https://www.edpsciences.org/en/maths-s2o-programme 